Combining Spatial and Parametric Working Memory in a Dynamic Neural Field Model

Weronika Wojtak, Stephen Coombes, Estela Bicho, Wolfram Erlhagen

IN: Artificial Neural Networks and Machine Learning - ICANN 2016

Volume 9886 of the series Lecture Notes in Computer Science, pp 411-418

Date: 13 August 2016

http://link.springer.com/chapter/10.1007/978-3-319-44778-0 48 


\title{
Combining spatial and parametric working memory in a dynamic neural field model
}

\author{
Weronika Wojtak ${ }^{1,3, \star}$, Stephen Coombes ${ }^{2}$, \\ Estela Bicho ${ }^{1}$, and Wolfram Erlhagen ${ }^{3}$ \\ 1 Research Centre Algoritmi, University of Minho, Portugal \\ \{w.wojtak, estela.bicho\}@dei.uminho.pt \\ 2 Centre for Mathematical Medicine and Biology, School of Mathematical Sciences, \\ University of Nottingham, UK \\ stephen.coombes@nottingham.ac.uk \\ 3 Research Centre for Mathematics, University of Minho, Portugal \\ wolfram.erlhagen@math.uminho.pt
}

\begin{abstract}
We present a novel dynamic neural field model consisting of two coupled fields of Amari-type which supports the existence of localized activity patterns or "bumps" with a continuum of amplitudes. Bump solutions have been used in the past to model spatial working memory. We apply the model to explain input-specific persistent activity that increases monotonically with the time integral of the input (parametric working memory). In numerical simulations of a multi-item memory task, we show that the model robustly memorizes the strength and/or duration of inputs. Moreover, and important for adaptive behavior in dynamic environments, the memory strength can be changed at any time by new behaviorally relevant information. A direct comparison of model behaviors shows that the 2-field model does not suffer the problems of the classical Amari model when the inputs are presented sequentially as opposed to simultaneously.
\end{abstract}

\section{Introduction}

A hallmark of higher brain function is the capacity to bridge gaps between sensation and action by maintaining goal-relevant information that is needed to perform a given task. Persistent neural activity which is commonly observed in prefrontal and association cortices is thought to represent a neural substrate for the accumulation and storage of information across time [11. Neurophysiological studies of persistent activity have frequently used a delayed response task in which the animal is required to remember a transient sensory stimulus (e.g., spatial location or frequency) across a short period to guide a rewarded response [15. To serve a working memory function, the internally sustained activity must be stimulus-selective so that the content of the memory can be decoded by downstream neural circuits. Neural discharge that varies according

\footnotetext{
* The work received financial support from the EU-FP7 ITN project NETT: Neural Engineering Transformative Technologies (no. 289146).
} 
to the value of continuous sensory or motor variables can be broadly classified in two distinct but not mutually exclusive coding schemes. Summation coding reflects the idea that parameter values are represented by a monotonic variation in neural firing rate 12. Place coding assumes a smooth bell-shaped tuning curve of individual neurons with a peak at a preferred value. At the population level, a specific parameter value is represented by a localized activity pattern in parametric space [5]. Depending on the specific coding scheme, stimulus-dependent persistent activity of neural populations has been classified as parametric or spatial working memory, respectively 15. While theoretical and experimental work has focused mainly on distinguishing both coding schemes based on optimality principles (e.g., accuracy of memorized sensory information), a more behavior-oriented perspective suggests that combining both types of memory representations might be advantageous for motor functions 13 . Imagine for instance a delayed response task in which the subject has to memorize the location of several stimuli, which, however, may differ in luminance contrast or the level of spatial attention directed to them. The memory strength of each item should reflect this additional information to bias, for instance, saccadic eye movements towards more salient stimulus locations.

In this paper, we present a novel dynamic field model that allows one to represent and memorize the integral of previous inputs in a robust manner. The framework of dynamic neural fields has been widely used in the past to model spatial working memory of continuous variables like position [27]1014]. The memory mechanism is based on the idea that a localized pattern of excitation (or "bump"), which is initially triggered by a brief input, can be sustained through strong recurrent excitatory and inhibitory connections within a neural population tuned to the continuous dimension. Since their level of abstraction favors analytical treatment [4, dynamic field models are also utilized for the development of new technical solutions inspired by neural processing principles 6. The two specific challenges we address in the present study are motivated by applications of a multi-item working memory [16]. The first question is concerned with the impact on the memory representation when multiple sensory events are presented sequentially as opposed to simultaneously. Since any existing bump in the field changes the initial condition for subsequent stimuli, it is not clear whether a stable multi-bump solution evolves in response to a series of sensory events even if the solution exists when the stimuli are presented simultaneously. The second question is more directly related to the suggested advantage of a combined spatial and parametric memory representation. Does the field dynamics support a simple monotonic relationship between the bump amplitude and the strength and/or duration of external stimuli 3]? In a similar vein, given a changing visual environment, can the internal representation be updated in the face of new input directed to a specific memory item ("retro-cuing" [8]). To answer these questions, we directly compare in numerical simulations the behavior of the classical Amari model [1] with the behavior of a new model consisting of two reciprocally coupled fields. 


\section{Model details}

The dynamics of the field model proposed and analyzed by Amari is governed by the following nonlinear integro-differential equation on a one-dimensional, spatially extended domain:

$$
\frac{\partial u(x, t)}{\partial t}=-u(x, t)+\int_{-\infty}^{\infty} w(|x-y|) f(u(y, t)-h) \mathrm{d} y+S(x, t),
$$

where $u(x, t)$ represents the activity at time $t$ of a neuron at field position $x$. In spatial working memory applications, neuron $x$ is assumed to be tuned to a continuous parameter (e.g., target direction). The function $w(|x-y|)$ denotes the distance-dependent strength of connections to neighboring neurons $y$. $S(x, t)$ represents a time-dependent localized input centered at site $x$, and $f(u-h)$ defines a firing rate function with threshold $h>0$ [1].

To simplify the analysis of pattern formation in his field model, Amari assumed $f(u)$ to be the Heaviside step function. In the present study, we use a smooth sigmoidal function with steepness parameter $\beta$, which approximates the Heaviside function for $\beta \rightarrow \infty$ :

$$
f(x)=\frac{1}{1+e^{-\beta(x-h)}} .
$$

Our novel model consists of two coupled fields, $u(x, t)$ and $v(x, t)$, governed by the two integro-differential equations

$$
\begin{gathered}
\frac{\partial u(x, t)}{\partial t}=-u(x, t)+v(x, t)+\int_{-\infty}^{\infty} w(|x-y|) f(u(y, t)-h) \mathrm{d} y+S(x, t), \\
\frac{\partial v(x, t)}{\partial t}=-v(x, t)+u(x, t)-\int_{-\infty}^{\infty} w(|x-y|) f(u(y, t)-h) \mathrm{d} y .
\end{gathered}
$$

Note that the neurons in field $v$ are driven by the summed activity from neurons in field $u$, but project their activity back locally only. For the coupling function $w(x)$, we follow Amari's original work and chose a Mexican-hat connectivity given by the difference of two Gaussian functions with a constant global inhibition:

$$
w(x)=A_{e x} e^{\left(-x^{2} / 2 \sigma_{e x}^{2}\right)}-A_{i n} e^{\left(-x^{2} / 2 \sigma_{i n}^{2}\right)}-g_{i n},
$$

where $A_{e x}>A_{\text {in }}>0$ and $\sigma_{i n}>\sigma_{e x}>0$ and $g_{i n}>0$.

Since the same coupling function is applied to the field $v$ with a negative sign, the shape of the synaptic strengths represents an inverted Mexican-hat, that is, inhibition dominates at shorter and excitation at longer distances.

To numerically approximate solutions of the continuum field models, we apply a forward Euler method with a sufficiently fine discretization mesh to equations (1) and (3). We assume a finite domain $\Omega$ of length $L=120$, which we discretize by dividing it into $N$ equal intervals of size $\Delta_{x}=0.005$. The chosen time interval $T=60$ is divided into $M$ equal steps of size $\Delta_{t}=0.01$. 
To compute the spatial convolution, we used the convolution theorem, stating that convolution in one domain equals point-wise multiplication in the other domain. The Fourier transform and the inverse Fourier transform were performed using MATLAB's in-built functions fft and ifft, respectively.

\section{Results}

In the following numerical examples, we consider an input distribution given by the sum of three equally spaced Gaussian functions

$$
S_{n b}(x)=\sum_{j=1}^{n} S_{s_{j}} e^{\left(-\left(x-x_{c_{j}}\right)^{2} / 2 \sigma_{s}^{2}\right)}-S_{i},
$$

centered at positions $x_{c_{j}} \in\{-40,0,40\}$. The parameter $S_{i}>0$ has been introduced to define a finite width of the positive input range. We use the same set of parameter values for both models to allow a direct comparison of results. These values are $\sigma_{s}=1.5$ and $S_{i}=1$ for the input, $A_{e x}=10, A_{i n}=3, \sigma_{e x}=2$, $\sigma_{i n}=3.5$ and $g_{i n}=1$ for the coupling function given by (4), and $\beta=1000$ for the firing rate function given by $(2)$. The strength $S_{s_{j}}$ and duration $d_{s_{j}}$ of the inputs are adjusted in the different examples as indicated in the figure captions.

Fig. 1 shows the evolution of a 3 -bump solution in response to the three inputs applied simultaneously at time $t=1$. The steady states of the field activity after cessation of the inputs indicate that both models support, in principle, the existence of multiple bumps, and thus, a multi-item working memory. However, the models behave quite differently when the same inputs are presented sequentially. As shown in the Fig. 2, the Amari model evolves a single bump whereas the 2-field model again converges to the 3-bump solution. In the Amari case, the steady state excitation pattern in response to the first input (which occupies the permitted total excitation length explained by the theory [1]) creates additional surround inhibition, which ultimately suppresses the initial excitation caused by the subsequent inputs. This is not the case for the 2-field model since the increased lateral inhibition in the $u$-field is compensated by positive feedback from the neurons in the $v$-field.

The results depicted in Fig. 3 demonstrate that in the Amari model, the relative timing of the inputs and their relative strength play a crucial role in a multi-item memory formation. If the temporal delay between inputs is sufficiently short so that excitation patterns triggered by previous inputs have not yet fully evolved, a multi-bump pattern may emerge (first row). Also, increasingly stronger inputs may compensate for the additional inhibition caused by existing bumps (second row). Importantly, since the bump shape is completely determined by the recurrent interactions, the input strength is not reflected in the bump amplitude. The 2-field model, on the other hand, shows a monotonic relationship as required by a parametric working memory that encodes analog parameters like for instance stimulus contrast in the firing rate. (Fig. 4, left). The dependency of bump amplitude on input strength is nearly linear for a 

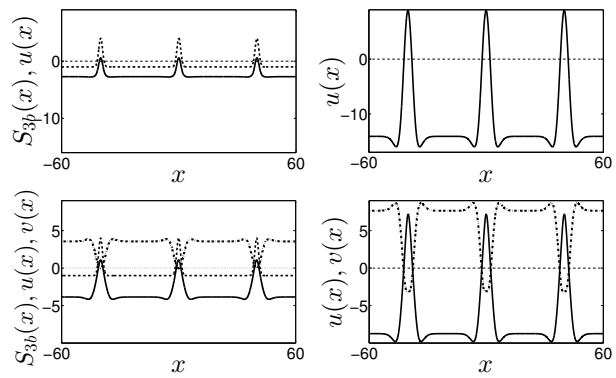

Fig. 1: Left: snapshot of the evolution of a 3-bump solutions at a time when the input distribution $S_{3 b}(x)$ (dashed line) is still present. Right: steady state solutions at time $t=60$. Top: activity $u(x)$ (solid line) of the Amari model. Bottom: activities $u(x)$ (solid line) and $v(x)$ (dashed-dotted line) of the 2-field model. Input parameters are $S_{s_{j}}=5$ and $d_{s_{j}}=1$.
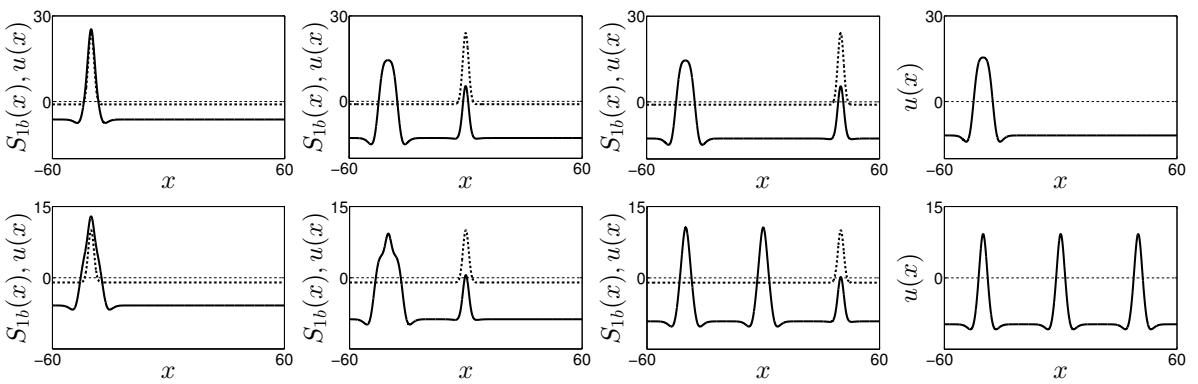

Fig. 2: Simulation of the field models with a sequence of three transient inputs $S_{1 b}(x)$ (dashed line). Top: activity $u(x)$ of the Amari model with inputs given by (5) with $S_{s_{j}}=25$ and $d_{s_{j}}=1$. Bottom: activity $u(x)$ of the 2-field model with inputs given by (5) with $S_{s_{j}}=11$ and $d_{s_{j}}=1$. The inputs were applied at times $t_{1}=1$ (first column), $t_{2}=17$ (second column) and $t_{3}=33$ (third column). The forth column shows the steady state solutions at time $t=60$.

very steep firing rate function, and becomes progressively more nonlinear with decreasing $\beta$ (right). The variation in the steepness of $f(u)$ over a relatively large parameter range shows that the encoding mechanism does not crucially depend on the fine tuning of parameters affecting the recurrent interactions. For a neural integrator to work properly, not only stimulus strength but also stimulus duration should matter. In the simulation presented in the Fig. 5, we study the influence of stimulus duration on the pattern formation. For the Amari model, as well as an increase of input strength also a prolonged input duration may overcome the additional inhibition caused by an already existing bump (left panel, compare with the simulation in Fig. 2). The 2-field model shows the same monotonic dependency of bump amplitude on duration (right panel) as for input strength (Fig. 4). In line with continuously changing task demands in dynamic 

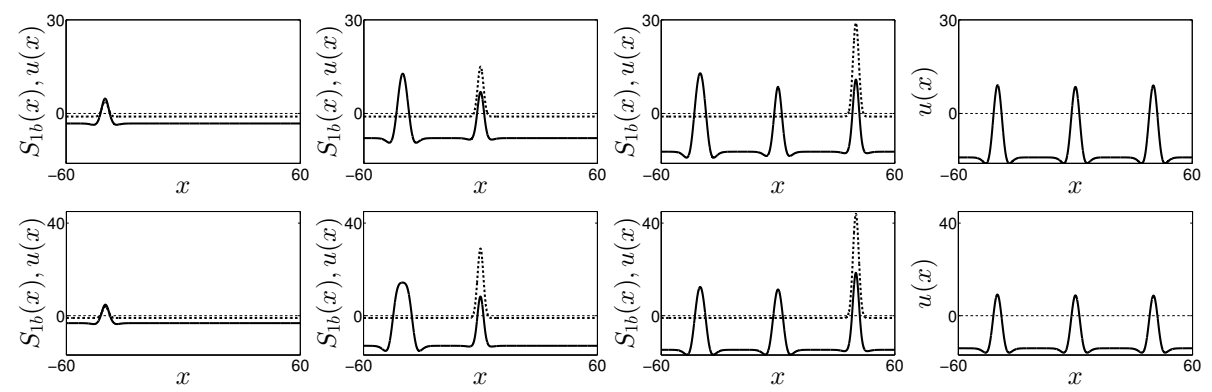

Fig. 3: Simulation of the Amari model with a sequence of three transient inputs $S_{1 b}(x)$ (dashed line). In the first row, the inputs were applied at times $t_{1}=1$ (first column), $t_{2}=2$ (second column) and $t_{3}=3$ (third column). In the second row, the inputs were applied at times $t_{1}=1$ (first column), $t_{2}=17$ (second column) and $t_{3}=33$ (third column). Input parameters are $S_{s_{j}} \in\{5,16,30\}$ (first row) and $S_{s_{j}} \in\{5,30,45\}$ (second row) and stimulus duration $d_{s_{j}}=1$. The fourth column shows the steady state solutions at time $t=60$.
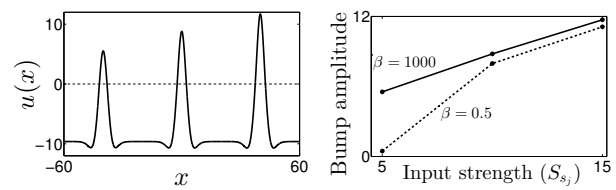

Fig. 4: Left: Steady state solution of the 2-field model with a firing rate function given by 2 with steepness parameter $\beta=1000$. A sequence of three inputs with different strengths $S_{s_{j}} \in\{5,10,15\}$ and equal duration $d_{s_{j}}=1$ was applied. Right: bump amplitude as a function of the input strength for two steepness parameter values, $\beta=1000$ and $\beta=0.5$.

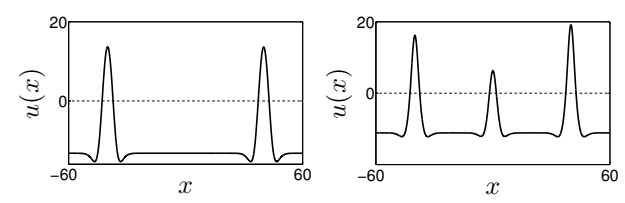

Fig. 5: Steady state solutions of the Amari model (left) and the 2-field model (right) triggered by a sequence of three inputs of different durations $d_{s_{j}} \in$ $\{2.5,1,3\}$. The inputs are given by (5) with $S_{s_{j}}=25$ (left) and $S_{s_{j}}=11$ (right), applied at times $t_{j} \in\{1,17,33\}$.

environments, converging experimental evidence indicate that top-down signals can prioritize items in working memory even after encoding [8]. For the working memory model this means that the bump amplitude should adapt to changing evidence at any time during the maintenance phase. Fig. 6] shows this ability in a model simulation in which a steady state activity pattern consisting of three 
bumps of equal strength (left) is updated by new inputs arriving at later times (right).

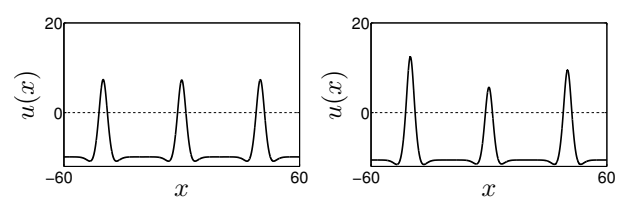

Fig. 6: Left: steady state solution of the 2-field model in response to a sequence of three inputs of equal strength $S_{s_{j}}=10$ presented at times $t_{j} \in\{1,2,3\}$. Right: steady state solution of the 2-field model after the presentation of additional inputs at position $x_{c_{4}}=-40$ (with strength $S_{s_{4}}=10$ ) and at position $x_{c_{5}}=40$ (with strength $S_{s_{5}}=5$ ). The inputs were applied for a duration $d_{s_{j}}=1$ at times $t_{4}=20$ and $t_{5}=22$, respectively.

\section{Discussion}

In this paper we have incorporated a second population into Amari's one-population neural field model of lateral inhibition type. The second population integrates the activity from the first population with an inverted Mexican-hat connectivity function and projects its activity back locally. We have shown in numerical simulations that the novel field model is able to explain input-selective persistent activity that increases monotonically with the time integral of the input. Since the sustained activity is spatially localized, the model combines the defining features of spatial and parametric working memory [15]. Moreover, the model supports a robust temporal integration of behaviorally relevant information over longer timescales.

Carroll and colleagues [3] have recently proposed a field model that also supports a continuum of possible bump amplitudes. Their model consists of separate excitatory and inhibitory populations that are intra- and interconnected with distance-dependent connectivity functions. However, the parameters of the network and the firing rate function (necessarily of piecewise linear shape) must be tuned precisely (see also [9]). In particular, the recurrent excitation must be inversely proportional to the slope of the nonlinearity to show a monotonic dependency of the bump amplitude on input strength. In contrast, the evidence of the present numerical study strongly suggests that the 2-field model is structurally stable to changes in model parameters. The lateral inhibition-type coupling function of the Amari model is known to support stable bumps over a whole range of parameter values [14, and significant changes in the shape of the firing rate function do not disturb parametric working memory (Fig. 4 .

Motivated by specific challenges in modeling multi-item memory with dynamic fields, we have also directly compared the behavior of the full 2-field 
model with the behavior of the $u$-population alone (Amari model). The results show that the feedback from the second population is necessary to ensure a robust formation of a multi-bump solution independent of whether the inputs are presented simultaneously or sequentially.

In future work, we plan to complement the numerical analysis of the novel field model with a more rigorous analysis of bump stability and the dependence of bump amplitude on the integral of the input.

\section{References}

1. S. Amari. Dynamics of pattern formation in lateral-inhibition type neural fields. Biological Cybernetics, 27(2):77-87, 1977.

2. M. Camperi and Xiao-Jing Wang. A model of visuospatial working memory in prefrontal cortex: recurrent network and cellular bistability. Journal of computational neuroscience, 5(4):383-405, 1998.

3. S. Carroll, K. Josić, and Z. P. Kilpatrick. Encoding certainty in bump attractors. Journal of Computational Neuroscience, pages 1-20, 2013.

4. S. Coombes. Waves, bumps, and patterns in neural field theories. Biological Cybernetics, 93(2):91-108, 2005.

5. W. Erlhagen, A. Bastian, D. Jancke, A. Riehle, and G. Schöner. The distribution of neuronal population activation (dpa) as a tool to study interaction and integration in cortical representations. Journal of neuroscience methods, 94(1):53-66, 1999.

6. W. Erlhagen and E. Bicho. The dynamic neural field approach to cognitive robotics. Journal of Neural Engineering, 3:36-54, 2006.

7. F. Ferreira, W. Erlhagen, and E. Bicho. Multi-bump solutions in a neural field model with external inputs. Physica D: Nonlinear Phenomena, 326:32-51, 2016.

8. I. C. Griffin and A. C. Nobre. Orienting attention to locations in internal representations. Cognitive Neuroscience, Journal of, 15(8):1176-1194, 2003.

9. A. A. Koulakov, S. Raghavachari, A. Kepecs, and J. E. Lisman. Model for a robust neural integrator. Nature neuroscience, 5(8):775-782, 2002.

10. C. R. Laing, W. C. Troy, B. Gutkin, and G. B. Ermentrout. Multiple bumps in a neuronal model of working memory. SIAM Journal on Applied Mathematics, 63(1):62-97, 2002.

11. E. K. Miller. The prefontral cortex and cognitive control. Nature reviews neuroscience, 1(1):59-65, 2000.

12. R. Romo, C. D. Brody, A. Hernández, and L. Lemus. Neuronal correlates of parametric working memory in the prefrontal cortex. Nature, 399(6735):470-473, 1999.

13. E. Salinas. How behavioral constraints may determine optimal sensory representations. PLoS Biol, 4(12):e387, 2006.

14. A. R. Schutte, J. P. Spencer, and Gregor Schöner. Testing the dynamic field theory: Working memory for locations becomes more spatially precise over development. Child development, 74(5):1393-1417, 2003.

15. Xiao-Jing Wang. Synaptic reverberation underlying mnemonic persistent activity. Trends in neurosciences, 24(8):455-463, 2001.

16. W. Wojtak, F. Ferreira, W. Erlhagen, and E. Bicho. Learning joint representations for order and timing of perceptual-motor sequences: a dynamic neural field approach. In Neural Networks (IJCNN), 2015 International Joint Conference on, pages 3082-3088. IEEE, 2015. 